\title{
ABHANDLUNG
}

\section{Gerechte Praxis statt universellem Standard. Eine alternative Perspektive auf Gerechtigkeit am Beispiel des globalen Drogendiskurses}

\author{
Eva Herschinger
}

No universal standard, just practice. An alternative perspective on justice and the global drug discourse

Abstract: Normative political theory has witnessed a renaissance in International Relations (IR), with the principle of justice and its meaning for the global sphere being prominently debated. Within these discussions, it is foregone conclusion that "poststructuralist approaches" have no normative potential. This paper takes issue with this conclusion and outlines how a poststructuralist approach to ethics can inform global politics. The paper elaborates an alternative reading of justice, arguing that justice is an empty normative signifier with universal aspiration aiming at discursive closure. To deal with closure, a just practice is necessary. It is a practice that constantly aspires to achieve justice while being aware of the impossibility of justice. The global discourse on drugs illustrates how an IR-poststructuralist approach yields insights in the possibility of ethical action in global politics.

Keywords: International relations, poststructuralism, justice, just practice, discourse on global drug prohibition.

Schlagwörter: Internationale Beziehungen, Gerechtigkeit, gerechte Praxis, Poststrukturalismus, globaler Drogendiskurs.

\section{Einleitung ${ }^{1}$}

Die Liberalisierung des Drogenkonsums, sagte Papst Franziskus anlässlich des Weltjugendtages 2013 in Rio de Janeiro, sei der falsche Weg, um dem Übel des Drogenhandels entgegenzutreten. Vielmehr, forderte er, müsse man die dahinterliegenden Probleme angehen, „indem man sich für mehr Gerechtigkeit einsetzt“ (Süddeutsche Zeitung Online 2010). Ein Jahr zuvor forderten die Initiatoren der Hanfparade mit dem Slogan „Freiheit, Gesundheit, Gerechtigkeit“ eine Legalisierung von Cannabis als Medizin, Rohstoff und Genussmittel (Hanfparade 2012).

1 Für hilfreiche Kommentare zu früheren Versionen dieses Beitrags möchte ich mich bei Jürgen Neyer und Frank Sauer, den Teilnehmer_innen des Panel „Internationale Gerechtigkeit“ der Tagung „Internationale Politische Theorie“ im Juni 2010 in Frankfurt sowie den Teilnehmer_innen des Treffens des AFK-Arbeitskreises „Theorie“ im Juli 2010 in Augsburg bedanken. 
2011 rief die National Association for the Advancement of Colored People (NAACP) die US-amerikanische Regierung dazu auf, den „Krieg gegen die Drogen zu beenden". NAACP-Vorstand Benjamin Todd Jealous erläuterte diese Forderung mit den Worten: „Heute hat die NAACP einen großen Schritt vorwärts getan, hin zu Gleichheit, Gerechtigkeit und effektiver Strafverfolgung “ (NAACP 2011).

Die Forderung nach Gerechtigkeit: Sie inspiriert nicht nur politische Theorien und philosophische Abhandlungen, sondern auch soziale Bewegungen, Politiker und Individuen gleichermaßen. Mehr noch, seit einiger Zeit erlebt Gerechtigkeit eine Renaissance als universale Rechtfertigung, als Letztbegründung für Forderungen, die den Anspruch auf „gerechte“ Verhältnisse erheben (Campbell 2011; Arneson 2006). So unterschiedlich diese Forderungen auch sein mögen - hier: Legalisierung statt Kriminalisierung von Drogen, Strafverfolgung statt militärische Maßnahmen, Bekämpfung der sozialen Ursachen statt Liberalisierung des Drogenkonsums -, es eint sie ihr Rekurs auf Gerechtigkeit als Begründung für die jeweilige Forderung. Diese Renaissance der Gerechtigkeit im politischen und gesellschaftlichen Diskurs findet ihren Widerhall - wie Andrew Hurrell es 2002 nannte - in einer der „bemerkenswertesten Entwicklungen der letzten fünfzehn Jahre in den Internationalen Beziehungen“ (IB) (Hurrell 2002, S. 138): die Wiederkehr der normativen politischen Theorie. Letztere sei heute wieder zu einer festen Größe in den IB geworden: „Research and writing on ethics and world politics has increased exceptionally" (Hurrell u. MacDonald 2013, S. 57). In der Tat, der Bedeutung von normativen Fragen für globale Politik wird - betrachtet man alleine die Literatur zu Gerechtigkeit - inzwischen eine deutlich höhere Aufmerksamkeit gewidmet. ${ }^{2}$ Auch im deutschsprachigen Kontext spiegelt sich diese Wiederkehr in dem unlängst geäußerten Ruf nach einer „Internationalen Politischen Theorie" wider (ZIB Forum 2010).

Dabei schimmert in diesen wissenschaftlichen Diskussionen ein sich hartnäckig haltender Vorwurf gegen die sogenannten poststrukturalistischen Ansätze ${ }^{3}$ in den IB durch. Es ist der Vorwurf eines „normativen Defizits“. Entweder können oder wollen Poststrukturalisten_innen sich den normativen Herausforderungen im Rahmen der normativen Trendwende nicht stellen - dieser Vorwurf, der vor allem in den 1990er Jahren vehement diskutiert wurde, ist inzwischen zu einer Art „Alltagsweisheit“ (Zehfuss 2013, S. 162) geronnen. Poststrukturalistische Ansätze seien trotz ihres de- und rekonstruktiven Potenzials außerstande, die bestehende Ordnung der Dinge zu evaluieren und zugunsten einer neuen, besseren Ordnung zu transzendieren. Vertreter_innen dieser Ansätze werden daher in der Diskussion um die Rolle von Gerechtigkeit und der Bedeutung normativer Theorie in den IB im Wesentlichen als Kritiker_innen anderer theoretischer Perspektiven zitiert.

Aus dem Schatten dieses Vorwurfs eines „normativen Defizits“ poststrukturalistischer IB-Ansätze will der vorliegende Beitrag „heraustreten““. Er macht sich

2 Notwendigerweise kann hier nur eine Auswahl aus der umfangreichen Literatur gegeben werden. Vgl. für jüngere Überblicke: Bell 2010; Frost 2009; Dryzek et al. 2006; für jüngere Schriften zu Gerechtigkeit: Hahn 2009; Forst 2007; Chwaszcza 2011; Hudson 2003; Miller 2007; Sen 2009.

3 Für eine Erläuterung des Labels „poststrukturalistisch“ siehe unten.

4 Abschnitt 2 erläutert, was unter Normativität im Kontext des „normativen Defizits“ verstanden wird. 
im Lichte der aktuellen Diskussionen nicht die Frage zu eigen, ob Poststrukturalisten_innen in der Lage sind, normative Erwägungen für globale Politik zu formulieren. Vielmehr geht es um die Frage, wie diese Erwägungen aus poststrukturalistischer Perspektive behandelt werden können. Da eine solche Frage für einen Beitrag dieses Formats entschieden zu umfassend ist, wird sich hier - wie die einführenden Zitate bereits vermuten ließen - einerseits auf das Prinzip der Gerechtigkeit konzentriert andererseits auf das Feld der internationalen Sicherheit, genauer auf die globale Drogenprohibition. Gerechtigkeit ist für das vorliegende Unterfangen besonders geeignet, da allgemein anerkannt wird, dass sie für die Stabilität und Akzeptanz globaler Ordnungen und spezifischer Politiken von hoher Bedeutung ist (Shapcott 2001; Goodin 2003). Weiterhin erscheint es höchst angemessen, dem Vorwurf des „normativen Defizits“ poststrukturalistischer IBAnsätze mit Blick auf die idée-clef moderner Politik entgegenzutreten und aufzuzeigen, dass die Ansätze zu mehr als nur zur kritischen Folie für andere Perspektiven taugen. Der Sicherheitsbereich ist als Illustrationsbeispiel einschlägig, da sich gerade in diesem Bereich seit dem Ende des Kalten Krieges die Frage nach Rechtfertigung und Kritik bestehender Formen politischer Ordnung drängender denn je stellt. Um eben jene Frage wird im globalen Drogendiskurs gestritten, d. h. ob eine Drogenpolitik gerecht ist, wenn bspw. Alkohol nicht unter die verbotenen Substanzen des Regimes der Vereinten Nationen (VN) fällt, das traditionelle Kauen von Coca-Blättern aber verboten ist, oder wenn der „Krieg gegen die Drogen“ zum Schutz vor Drogenkriminalität geführt wird, er aber zu mehr Kriminalität und Menschenrechtsverletzungen bei den schwächsten Glieder der Drogenhandelskette ${ }^{5}$ führt.

Gerechtigkeit aus poststrukturalistischer Sicht, so das zentrale theoretische Argument des Beitrags, ist ein leerer normativer Signifikant, der mit universellem Anspruch auftretend eine diskursive Schließung anstrebt. Diese Schließung ist jedoch aufgrund der Vielzahl partikularer Gerechtigkeitsvorstellungen in der heutigen internationalen Politik unmöglich - ein Umstand, der in der zum Klischee geronnen Feststellung, dass Gerechtigkeit ein „essentially contested concept“ (Gallie 1956) sei, eingefangen ist. Die Unmöglichkeit einer vollkommenen Schließung liegt nicht zuletzt darin begründet, dass Gerechtigkeit die positive Umkehrung einer negativ erfahrenen Situation ist. Der anzustrebende Umgang angesichts dieser Schließungen, so argumentiert der Beitrag weiter, ist eine Diskurspraxis des Entscheidens, die Gerechtigkeit zwar anstrebt, die Unmöglichkeit ihrer endgültigen Erreichung aufgrund ihrer beständigen Zukünftigkeit aber reflexiv mit einbezieht. Es geht also um eine gerechte Praxis und weniger um (die Fixierung von) Gerechtigkeit.

Dieses Argument soll im Folgenden im Rückgriff auf zentrale Arbeiten von poststrukturalistischen IB-Autor_innen sowie von Jacques Derrida, Ernesto Laclau und Chantal Mouffe entwickelt werden. Die Bedeutung des Arguments

5 Als die „schwächsten Glieder der Drogenhandelskette“ werden vor allem die Drogenkonsument_ innen, Kleinkurier_innen und die ländliche Bevölkerung in Zonen mit illegalen Anpflanzungen bezeichnet. Vgl. das Statement der European Coalition for Just and Effective Drug Policies (1998). 
von Gerechtigkeit als leerer normativer Signifikant und das Anstreben einer gerechten Praxis wird mit Blick auf den globalen Drogendiskurs illustriert, innerhalb dessen das globale Drogenprohibitionsregime (entstanden von 1961 bis 1988 unter der Ägide der Vereinten Nationen) eine besondere Rolle spielt. Das Ziel des Beitrags ist allerdings zuvörderst ein theoretisches, d. h. zu erarbeiten, wie Gerechtigkeit in den IB poststrukturalistisch gelesen werden kann. So zeigt der Beitrag auf, warum es kein „normatives Defizit“ poststrukturalistischer Ansätze in den IB gibt, sondern der Vorwurf vor allem der Privilegierung einer bestimmten Vorstellung davon, wie normative Fragen erörtert werden sollen, geschuldet ist.

Zunächst wird dazu die Attestierung des „normativen Defizits“ poststrukturalistischer Ansätze in den IB diskutiert (Abschnitt 2). Wie poststrukturalistische Forscher_innen in den IB bislang mit normativen Fragen globaler Politik umgegangen sind und wie sich das Argument des Beitrags hier verortet, legt Abschnitt 3 dar. Es folgt die Entwicklung des zentralen Arguments von Gerechtigkeit als leerem normativen Signifikanten und der Relevanz der gerechten Praxis (Abschnitt 4). Der fünfte Abschnitt führt die Illustration des Arguments anhand der Betrachtung des Drogendiskurses, hier vor allem des globalen Drogenprohibitionsregimes, zusammen. Das Fazit kontextualisiert die gewonnen Einsichten und reflektiert diese im Lichte des vermeintlichen „normativen Defizits“.

Zwei Einschränkungen seien an dieser Stelle geäußert: Erstens, dieser Beitrag ist der Versuch einer theoretischen Auslotung (nicht zuletzt deshalb ist die empirische Illustration auch nur als Illustration gemeint). Er will neue Denkanstöße vermitteln. Insofern ist der Beitrag ein explorativer Vorschlag, wie sich normative Fragen aus poststrukturalistischer Sicht in einem bedeutsamen Feld wie Sicherheit für die IB konzeptualisieren lassen und welche Implikationen sich daraus ergeben. Zweitens stellt sich die Frage des Labels. Eingangs wurde von den „sogenannten poststrukturalistischen Ansätzen“ in den IB gesprochen. Zweifelsohne ist es wenig leserfreundlich, durchgängig ein „sogenannt“ vorzuschieben. Im Rahmen diese Beitrags soll die Bezeichnung „poststrukturalistische Ansätze in den IB“ jedoch bewusst genutzt werden, ganz im Sinne der Autor_innen, die sich dieses Labels bedienen (Der Derian u. Shapiro 1989; Ashley u. Walker 1990; George 1994; Hansen 2006; Zehfuss 2013). ${ }^{6}$ Dabei wird der Begriff Poststrukturalismus verstanden als eine „broad church, as a label covering a variety of intellectual trends, ranging from Foucault's analysis of discursive formations, to Lacanian psychoanalysis, Derrida's deconstruction and Barthe’s semiology“ (Laclau 2000a, S. x).

\section{Die IB, der Poststrukturalismus und die Rückkehr normativer politischer Theorie}

Wie eingangs erwähnt, erlebt die Disziplin der IB seit mehr als zwei Jahrzehnten eine sich intensivierende Debatte über normative Fragen. Die Gründe für diese Wiederkehr sind vielfältig. Internationale Politik war zunehmend von ethnischen,

6 Ich habe diese Bezeichnung auch in früheren Arbeiten mit ähnlicher Begründung gewählt (Herschinger 2011, 2012). 
nationalistischen Konflikten, dem Ruf nach (humanitären) Interventionen wie nach einer Stärkung der VN oder der Klage über die wachsende globale Ungleichheit geprägt. Das grelle Licht der post-1990er Jahre legte die moralischen Dilemmata der Disziplin offen und schien nach einer Einbindung von übergeordneten Fragen, wie sie die politische Theorie stellt, zu verlangen.

Dabei ist zunächst von Bedeutung, dass die Diskussion, die fast ausschließlich im englischsprachigen Raum geführt wurde, sich von Anfang an weniger auf den Begriff des Normativen konzentrierte, sondern direkt auf die Frage nach der Möglichkeit eines „ethical turn in IR“ (Frost 1998) - kurz: Die Frage nach dem Normativen wurde im Wesentlichen als ethische Frage verstanden. Der Frage, ob die IB das Potenzial habe, ethischen Belangen gerecht zu werden, wurde Ende der 1990er Jahre noch mit Zweifeln begegnet. Denn gerade vor der zentralen Frage nach dem Wesen ethischen Verhaltens - dem „Was sollen wir tun?“ - schreckte die IB weiterhin zurück. Dies gelte vor allem für die poststrukturalistischen Ansätze, seien sie doch mit Blick auf normative Fragen in der internationalen Politik letztlich „a turning away from both political responsibilities and a concern for suffering“ (Lyon 1999, S. 103; ähnlich Frost 1998, S. 129; Brown 1992, S. 223). Angesichts der Vielzahl an theoretischen Strömungen, die, in der Regel aus der benachbarten politischen Theorie stammend, sich in der breiteren IB inzwischen mit ethischen Fragen beschäftigen - angeleitet von Kosmopolitanismus über Kommunitarismus hin zu Kritischer Theorie, um nur die prominentesten Kandidaten zu nennen - kann man diese allgemeinen Zweifel gegenüber dem normativen Potenzial der IB heute nicht mehr aufrechterhalten. Denn Überlegungen zu normativen Fragen aus den genannten theoretischen Gebäuden inspirieren heute zahlreiche Perspektiven auf zeitgenössische globale Politik, sei es Außenpolitik, Menschenrechte, Konfliktbewältigung oder Umweltpolitik.

Die genannten Theorien unterscheiden sich allerdings in einem wesentlichen Punkt von poststrukturalistischen Ansätzen in den IB: Sie liefern eine Entscheidungsgrundlage, um zwischen unterschiedlichen Forderungen nach bspw. Gerechtigkeit diejenigen herauszufinden, die als gerechter gelten können und daher erfüllt werden sollten. Auch der Vorwurf des „normativen Defizits“ hat hier seinen Ursprung: Verbirgt sich hinter ihm doch eine ganz bestimmte Lesart von Normativität. Nämlich jene, die nach dem Wesen des Normativen fragt und es in rationalen Gründen findet, denn zur „Erklärung der Normativität muss es demnach einen [...] überzeugenden Grund für das ,Sollen' geben“ (Gosepath 2009, S. 256; vgl. auchWallace 2011; Forst 2004). „,Normativ“ bedeutet in grober Annäherung so viel wie den Status eines Maßstabes zu haben, gemessen an dem etwas richtig oder falsch, gut oder schlecht, zulässig oder unzulässig, angemessen oder unangemessen ist" (Gosepath 2009, S. 251). Dieses Verständnis von Normativität lag und liegt quer mit dem Anliegen poststrukturalistischer Ansätze in den IB (siehe dazu weiter unten).

Allerdings schienen prominente Poststrukturalist_innen dem Vorwurf des „normativen Defizits“ Vorschub zu leisten, wenn sie sich der Annahme von universalen Standards verweigerten, an denen rivalisierende Ansprüche, Ansichten und Verhaltensweisen gemessen und somit als gerecht oder ungerecht, als legitim oder illegitim angesehen werden könnten (vgl. vor allem Ashley und Walker 
1990). In dieser Verknüpfung liegt vermutlich die wesentliche Ursache dafür, dass der Vorwurf des „normativen Defizits“ aus den 1990er Jahren für poststrukturalistische Ansätze weiterhin erhoben wird. Sie würden nur Theoretisches über internationale Politik produzieren, ohne normative und politische Probleme der realen Welt zu adressieren. Alles in allem scheint das „normative Defizit“ poststrukturalistischer Ansätze also weiterhin eine beschlossene Sache (Brown 1992, 1994; Cochran 1995; White 1988; Fagan 2013).

Trotzdem - oder gerade deswegen - wird die Frage, ob, und wenn ja wie, Poststrukturalist_innen fruchtbare normative Überlegungen zur globalen Politik äußern könnten, in den letzten Jahren in den IB wieder diskutiert (Smith 2004; Jabri 2007; Engelkamp et al. 2012; Fagan 2013). Es erscheint daher mehr als gerechtfertigt, die oben skizzierte Einschätzung im Lichte der im letzten Jahrzehnt entstandenen Forschung hier differenzierter zu betrachten. Dies gerade auch vor dem Hintergrund, dass sich jüngere Stimmen in der deutschen Debatte poststrukturalistische Ansätze als mögliche „Fluchtwege“ (Deitelhoff 2010, S. 287) aus den konsensorientierten Global Governance Modellen internationaler Politik vorstellen können oder auf dieser Basis das Programm einer kritischen Normenforschung entwerfen (Engelkamp et al. 2012, S. 110-122). Da Poststrukturalisten_innen Konflikte als ontologische Bedingung des Politischen betrachten, sind sie sensibler für Widerstand und Kontestation, also jene sozialen Prozesse, die das zeitgenössische globale Geschehen verstärkt prägen (Mouffe 1993).

Zunächst könnte man sich aus poststrukturalistischer Perspektive natürlich auf den Standpunkt stellen, dass es bei der Diagnose des „normativen Defizits“ um den Versuch der Übertragung eines hegemonialen Verständnisses von Normativität geht; sprich um die Disziplinierung poststrukturalistischer Ansätze im Foucaultschen Sinne. Das ist möglich. Hier soll aber ausgeführt werden, wie sich aus poststrukturalistischer Perspektive mit normativen Fragen beschäftigt werden kann, wie in einer Zahl von IB-Schriften in den letzten zehn Jahren geschehen (siehe folgenden Abschnitt). Dabei zeigt sich, dass die „Alltagsweisheit“ des „normativen Defizits“ vor allem ein Problem der Kritiker_innen poststrukturalistischer IB-Ansätze zu sein scheint, geht es ihnen doch in den meisten Fällen darum, einen universalen normativen Standard zunächst zu definieren, eine Entscheidungsgrundlage zu begründen und von dort aus eine bestimmte Politik zu entwickeln, die im Sinne dieses Standards als normativ angemessen zu bezeichnen ist (Critchley 1992; Campbell 1998a; Fagan 2013). Wie schon erwähnt, die Etablierung universeller normativer Prinzipien ist den Poststrukturalisten_innen fremd. Wenn der Schluss daraus aber eben nicht lautet, dass die Ansätze nicht in der Lage sind, normative Überlegungen $\mathrm{zu}$ formulieren und somit zu globaler Politik nichts sagen zu können, dann muss der Schluss die Frage nach dem „Wie dann?“ sein, d. h. wie normative Erwägungen für globale Politik aus poststrukturalistischer Perspektive behandelt werden können.

Dazu ist es notwendig, sich alternativen Verständnissen von Normativität zuzuwenden, wie sie von poststrukturalistischen Autor_innen favorisiert werden. So hat gerade Derrida - auf den sich neben Michel Foucault das Gros der Poststrukturalist_innen in den IB bezieht - eine alternative Variante zur begründungstheoretischen Sicht auf Normativität entwickelt. Seine Position sucht die Fundamente 
einer solchen Normativität zu erschüttern und Normativität „nicht als festhaltbares Proprium, sondern als kritische Aufforderung zur Selbstbefragung “ (FlügelMartinsen 2009, S. 10, 24) zu verstehen. Das heißt, Normativität ist die kritische Befragung herrschender normativer Prinzipien, der von ihnen etablierten Maßstäbe und Standards, ein Infragestellen und Kritisieren, das so „zugleich den mit [ihnen, EH] verbundenen Geltungsanspruch ins Rutschen“ (Flügel-Martinsen 2010, S. 152) bringt.

Allein schon im Rückgriff auf dieses alternative Verständnis von Normativität verwirft dieser Beitrag den Vorwurf des „normativen Defizits“, privilegiert er doch erstens die eine Lesart von Normativität als auf Gründen aufruhend und verneint damit das Potenzial anderer Ansätze, normative gehaltvolle Aussagen treffen zu können. Wie mit den Konsequenzen solch alternativer Normativitätsbegriffen wie eben jenem der ,Befragung' für die Analyse internationaler Politik umgegangen werden kann, dazu will der vorliegende Beitrag anhand des Prinzips der Gerechtigkeit nachstehend Anregungen liefern.

Zweitens gibt es aus poststrukturalistischer Perspektive gar keine Möglichkeit, ethischen Fragen zu entgehen, da (globales) Leben unausweichlich ethisch ist (Pin-Fat 2009, S. 44; Campbell u. Shapiro 1999b; Lawler 2008). Mit der Verwendung des Begriffes „ethisch“ soll hier jedoch nicht die eben abgelehnte begründungstheoretische Lesart von Normativität durch die Hintertür wieder eingeführt werden. Vielmehr wird damit der - führenden - englischsprachigen Debatte um die Frage nach dem „normativen Defizit“ Rechnung getragen, in der gerade auch Poststrukturalist_innen, Normativität im Wesentlichen unter dem Begriff der Ethik abhandeln (Bulley 2006; Burke 2013; Campbell 1993; Campbell u. Shapiro 1999b; Fagan 2013; George 1995; Pin-Fat 2010). ${ }^{7}$ Zugleich trifft, was mit Blick auf die Lesart von Normativität gesagt wurde, auch auf das poststrukturalistische Verständnis von Ethik zu. Kennzeichnend für Letzteres ist, dass Ethik im herkömmlichen Sinne als problematisch verstanden wurde, denn diese beruhe auf

the imposition and observance of limitation that can themselves be unambiguously defined and justified from the standpoint of a sovereign center of judgment that commands a space, authenticates what is valuable and good within, defines alien and dangerous modes of thought and conduct to be excluded, and is itself beyond question" (Ashley u. Walker 1990, S. 390).

Es geht also erneut nicht darum, die Voraussetzungen und Kriterien für begründbares ethisches Verhalten auf Basis eines normativen Standards zu postulieren, sondern durch die Einsicht, dass das Ethische politisch ist und das Politische ethisch - es hier also keine Trennung geben kann - antifundationalistische Lesarten von Ethik und letztlich auch von Normativität zu ermöglichen, kann doch weder das Politische noch das Ethische von vorneherein feststehen, sondern durch das Verständnis von Normativität als einer Befragung und Entgründung wird die Offenheit von beidem ermöglicht (Flügel-Martinsen 2009, S. 263).

7 Der Beitrag adressiert insofern auch die dominante englischsprachigen Debatte in den IB, da in ihr die wesentlichen Einwände gegen den Vorwurf des „normativen Defizits“ formuliert wurden. 


\section{Normative Fragen globaler Politik aus poststrukturalistischer Perspektive}

Poststrukturalist_innen in den IB entwickelten ihre Verständnisse von Normativität (und im englischsprachigen Kontext von Ethik) aus einer spezifischen erkenntnistheoretischen Position heraus. Sie offerierten Wege, die Welt, in der wir leben, nicht als objektiv gegebene (unveränderbare) „Realität“ hinzunehmen; sie erlaubten es, zu hinterfragen, wie unser Wissen über eben diese Welt entsteht, indem sie die Ansicht ernst nahmen, dass Forscher_innen stets Teil der Welt ihrer „Forschungsobjekte“ sind. Selbst wenn Verallgemeinerungen hier nicht unproblematisch sind, so geht es Poststrukturalisten_innen generell um die Fragen danach, wie Repräsentationen der Welt zustandekommen und wie sie unweigerlich scheitern, welche sich verändernden sozialen Praktiken die globale Politik prägen, welche Machtverhältnisse dadurch (bspw. durch Subjektivierungen) (re)produziert werden und welche Rolle Individuen (bspw. durch Widerstand gegen Subjektivierungen) in der globalen Politik spielen. Kurz, es geht darum, die Kontingenz dessen, was gegeben, was real zu sein scheint, aufzuzeigen, um alternative Denkweisen über die Welt und die Rolle der Forschenden darin zu ermöglichen. Poststrukturalismus ,reveals the politics involved in producing reality as commonsensical or even inevitable“ (Zehfuss 2013, S. 152).

Nicht zuletzt dadurch ist es unmöglich - wie bereits oben ausgeführt -, die politische Dimension von der ethischen zu trennen, mehr noch: „[T]he moment ethical questions are separated out, things have already gone wrong“ (Zehfuss 2013, S. 162). Gerade dieser Anspruch, die politische Dimension von Realitätskonstruktionen in den Blick zu bekommen, führte Poststrukturalisten_innen in den IB - eingedenk der Annahme, dass „ethics is all around us and is unavoidable“ (Pin-Fat 2009, S. 44) - zur Haltung eines „,ethos of political criticism“ (Campbell 1998a, S. 4) oder zu einem „ethos of critique“ (Jabri 2007, S. 167). Wie genau ein solches Ethos und ein damit korrespondierendes Verständnis von Normativität aussehen könnten, wurde in der poststrukturalistischen IB-Literatur vielfältig diskutiert.

Grob skizziert lassen sich drei Sichtweisen unterscheiden, die sich jedoch nicht gegenseitig ausschließen, sondern jeweils verschiedene Schwerpunkte formulieren. Erstens sehen sich einige poststrukturalistische Autoren_innen einer emanzipatorischen Agenda verpflichtet. So argumentierte Jim George: „[T]here are no ,good“ reasons why others in the world should not have the opportunities that I have had $[\ldots]$ “ und „[A] critical ethics would be one which supports political strategies which seek to provide this opportunity and, in general, opposes political strategies which seek to deny it“ (George 1995, S. 219). Insofern könne die Unterscheidung zwischen „guten“ und „schlechten“ Anderen als Rechtfertigung für Widerstand dienen (Couzens Hoy 2004; Kearney 2003).

Autoren wie Michael Dillon oder Richard Ashley stehen für eine zweite Position. Nach beiden gilt es vor allem, sich bewusst zu machen, dass unser Dasein immer ein zerrissenes ist, sich selbst fremd. Das verlange nach einem „ethos that welcomes rather than denies the human plurality that is integral to its being “ (Dillon 2013, S. 101), nach einer „ethics of marginal conduct“ sowie nach einer „ethics of freedom“ (Ashley u. Walker 1990, S. 392, 395). 
Eine dritte Sicht auf die Frage des kritischen Ethos - die vor allem in der jüngeren IB-Literatur an Raum gewonnen hat - stellt das Konzept der Differenz ins Zentrum der Analysen (Edkins 1999; Pin-Fat 2010; Vaughn-Williams 2009; Zehfuss 2011). Was die drei Positionen eint, ist das oben schon angesprochene „Nein“ zu universalen ethischen Prinzipen, vielmehr sei „[e]thics [...] an ongoing historical practice“ (Walker 1993, S. 51). Dazu kommt ein Verständnis von Ethik als relational, bzw. von einer ethischen Bindung an und Verantwortung für den Anderen (Campbell u. Shapiro 1999a, S. xi).

Gerechtigkeit spielt in diesen Debatten keine prominente Rolle (eine Ausnahme ist Shapiro 2012); sie ist eher implizit Thema, entweder im Kontext von der Reund/oder Dekonstruktion zentraler IB- sowie kontinentalphilosophischer Texte oder als Teil empirischer Analysen (Campbell 1998b; 1999; Edkins 2003, S. 226; Fagan 2013; Pin-Fat 2010; Walker 1993, 2010). Einen zentralen Grund für diese Zurückhaltung formulierten David Campbell und Michael J. Shapiro schon vor geraumer Zeit: „[A] striving for the rules and principles of justice, especially those that demand impartially, effects injustice" (Campbell u. Shapiro 1999a, S. xi). Dieser Einschätzung und der Gefahr, die sie aufzeigt, ist zuzustimmen. Jedoch argumentiert dieser Beitrag, dass es einer poststrukturalistischen Sicht auf Gerechtigkeit in den IB bedarf - gerade mit Blick auf die bereits erwähnte zunehmende Bedeutung von Gerechtigkeit als „Letztrechtfertigung“ für höchst unterschiedliche Forderungen in der internationalen Politik und angesichts einer weitgehenden Übereinstimmung in der Literatur der IB und der politischen Theorie bezüglich der enormen Bedeutung von Gerechtigkeit für die Akzeptanz und Stabilität globaler Ordnungen (Welch 1993, S. 1; Forst u. Günther 2011, S. 11; vgl. auch Shapcott 2001; Goodin 2003). Denn eine poststrukturalistische Sicht auf Gerechtigkeit kann ihre Umstrittenheit nicht nur als wissenschaftlichen Allgemeinplatz benennen. Vielmehr liegt in der Idee von Konflikt - um die Bedeutung von dem, was gerecht sein soll - als ontologische Bedingung des Politischen der Ausgangspunkt. Die Umstrittenheit wird so nicht zum „Problem“, aus dem ein Kern von Gerechtigkeit herausgefiltert werden muss und somit letztlich eine partikulare Vorstellung von Gerechtigkeit zur allgemeingültigen erhoben wird. Stattdessen können die Machtverhältnisse, die sich in den Fixierungen der Bedeutung von Gerechtigkeit zeigen, auf diese Weise offengelegt werden. Insofern kann eine poststrukturalistische Sicht aufgrund ihrer Reflexivität die Gefahr, die in den Fixierungsversuchen von Gerechtigkeit liegt - sprich, mehr Ungerechtigkeit zu produzieren - systematisch reflektieren und die Implikationen einer Bindung an und Verantwortung für den Anderen adressieren. Letztlich liegt hier der doppelte Beitrag dieses Papiers: Zum einen wird Gerechtigkeit erstmals in den Fokus poststrukturalistischer IB-Überlegungen zu Normativität und einem kritischen Ethos gerückt; zum anderen wird Gerechtigkeit, als eines der Lieblingskinder normativer, internationaler politischer Theorie dazu geradezu ideal geeignet, für eine Widerlegung des Vorwurfs vom „normativen Defizit“ poststrukturalistischer Ansätze in den IB genutzt. Dafür ist aber zunächst die Erarbeitung eines poststrukturalistischen Verständnisses von Gerechtigkeit erforderlich, das, gemäß des Arguments des Beitrags, Gerechtigkeit als leeren normativen Signifikanten konzeptualisiert, die durch eine Diskurspraxis des Entscheidens (gerechte Praxis) an- 
gestrebt werden kann (und soll), die Unmöglichkeit ihrer endgültigen Erreichung aber reflexiv einbezieht.

\section{Gerechtigkeit poststrukturalistisch gelesen}

Die obigen Ausführungen haben bereits einige zentrale Einsichten poststrukturalistischer Ansätze angedeutet. Ganz in der Tradition poststrukturalistischer Arbeiten in den IB greift der Beitrag für seine Lesart von Gerechtigkeit auf einschlägige Theoretiker_innen zurück, zum einen Jacques Derrida, zum anderen Ernesto Laclau und Chantal Mouffe. ${ }^{8}$ Mit Gesetzeskraft. Der „mythische“ Grund der Autorität (1991) beschäftigte sich Derrida explizit mit Gerechtigkeit im Rahmen von Dekonstruktion - eine Beschäftigung, die entscheidende Wege für eine poststrukturalistische Lesart von Gerechtigkeit für die IB aufzeigt. ${ }^{9}$ Und die von Laclau und Mouffe entworfene Hegemonietheorie erlaubt einerseits diskursive Schließungen zu analysieren, d. h. wie die Durchsetzung bestimmter Gerechtigkeitsvorstellungen im Diskurs funktionieren; andererseits erlaubt ihr Diskursbegriff die Einbindung von Praktiken, ein Umstand, der für das Argument einer gerechten Praxis entscheidend ist.

Poststrukturalistische IB-Ansätze betonen die Bedeutung von Sprache, Diskurs und Kontingenz in der Betrachtung von internationaler Politik. Sprache ist ontologisch bedeutsam und produktiv; Realität ist nicht „objektiv“ erfahrbar, denn Objekte sind nur in einem sprachlich vermittelten Kontext verstehbar. Vielmehr - und dies lässt sich trotz unterschiedlicher Diskursbegriffe in der poststrukturalistischen (IB-)Literatur festhalten - fungiert der diskursive Kontext als Horizont für die Konstituierung von Objekten und Subjekten, deren Bedeutung wiederum auf einem sozial konstruierten System von Regeln und Differenzen beruht (Howarth u. Stavrakakis 2000, S. 3). ${ }^{10}$ So können mit der Hegemonietheorie von Laclau und Mouffe Diskurse als eine „structured totality“ (Laclau u. Mouffe 2001, S. 105) verstanden werden, als Systeme von Praktiken, die Subjekte und Objekte so miteinander in Beziehung setzen, dass sie Bedeutung erlangen. Diskursive Strukturen sind also keine kognitiven Einheiten, sondern Praktiken der Artikulation, die soziale Verbindungen und Zusammenhänge begründen und organisieren.

Diskurs als eine „Totalität“ zu denken, sollte nicht missverstanden werden. Diskurse sind eine von Kontingenz und Temporalität gekennzeichnete Struktur, von Brüchen und Rissen durchsetzt, da die Beziehungen der durch den Diskurs verbundenen Differenzen und ihre Bedeutung sich beständig wandeln können. Dies erklärt sich aus dem Sprachverständnis, auf dem wiederum der Diskursbegriff aufbaut. Sprache ist einerseits strukturiert, da sie ein System differentieller

8 Für eine kleine Auswahl von Derrida sowie von Laclau und Mouffe inspirierten IB-Studien siehe: Campbell 1998a; Dillon 2013; Bonacker 2002; Hansen 2006; Herschinger 2011; Renner 2013; Methmann 2010. Im deutschsprachigen Kontext sind vor allem die Arbeiten von Thomas Diez (1999) und Martin Nonhoff (2006) hervorzuheben.

9 Speziell Michael Dillon (1999) hat sich mit diesem Werk in den IB beschäftigt.

10 Wenn es auch keine objektive Bedeutung jenseits von sprachlichen Repräsentationen gibt, so heißt dies nicht, dass die Materialität von bspw. einem Tisch nicht existiert (Laclau u. Mouffe 2001, S. 108). 
Zeichen ist, deren Bedeutung durch Kombination oder Subsumption von Artikulationen entsteht (Derrida 1976). Artikulation organisiert Sprache, da eine artikulatorische Praxis bestimmte Signifikanten und Signifikate miteinander verbindet und ihnen so eine instabile, temporäre Bedeutung verleiht (Laclau u. Mouffe 2001, S. 113). Unterschiedliche Anordnungen sind möglich, denn ein Zeichen besteht aus einem Signifikanten (die Buchstaben auf einer Buchseite oder der Ton, der in unserem Ohr erklingt) und einem Signifikat (das Konzept, das vor unserem geistigen Auge erscheint, wenn wir den Signifikanten lesen oder hören). Andererseits ist Sprache instabil, da ein Signifikant in einem endlosen Prozess auf unterschiedliche Signifikate verweist, ohne jemals eine letztgültige Bedeutung zu erlangen. Die Anordnung bzw. die Artikulation unterschiedlicher Signifikate und Signifikanten ist insofern kontingent (Glynos u. Howarth 2007, S. 179).

Das bedeutet, dass der Versuch, Bedeutung letztgültig festzuzurren, Diskurse also zu schließen, zum Scheitern verurteilt ist: „, [N]either absolute fixity nor absolute non-fixity is possible“ (Laclau u. Mouffe 2001, S. 111). Eine Festschreibung ist unmöglich, denn laut Derrida wird es immer eine überbordende Instabilität geben, die zu einer Unentscheidbarkeit einer jeden Äußerung führt. Ein Wort alleine ist nicht in der Lage, eine ganz bestimmte Bedeutung vollständig hervorzurufen, diese kann nur mit Hilfe weiterer Worte erklärt und definiert werden (was Derrida mit seinem Neologismus différance umschrieb). Gleichzeitig kommt es beständig zu Schließungsbestrebungen, da jeder Diskurs auch der machtgeladene Versuch ist, Bedeutungen so lange wie möglich auf die eine, aber nicht die andere Weise festzuschreiben. So werden durch partielle Fixierung Identitäten und soziale Formationen temporär möglich. Wirft man exemplarisch einen Blick auf den globalen Drogendiskurs, so bedeutet eine solche Konzeptualisierung von Sprache, Diskurs und Kontingenz einerseits, dass der „Krieg gegen die Drogen“ auf spezifischen, kontingenten Repräsentationen des Sicherheitsproblems „Drogen“ beruht, das er bekämpfen will. Andererseits ermöglichen diese Repräsentationen die jeweiligen Gegenmaßnahmen wie bspw. Erntevernichtungsprogramme, indem sie diese verstehbar und akzeptabel machen.

Vor diesem Hintergrund ergibt sich eine spezifisch poststrukturalistische Lesart des Prinzips der Gerechtigkeit. In den eingangs zitierten Forderungen nach Legalisierung, einem Ende des „Kriegs gegen die Drogen“ oder einem Nein zur Liberalisierung diente Gerechtigkeit den unterschiedlichsten Akteuren als Basis für die Rechtfertigung ihrer Forderungen. Aus poststrukturalistischer Sicht führen die Widersprüche, die sich aus diesen vielfachen Verwendungen für die Gerechtigkeit ergeben, zu Entleerung und Fragmentierung des Signifikanten Gerechtigkeit - und damit letztlich auch zur Unmöglichkeit seiner Realisierung: Gerechtigkeit ist, so das Argument des Beitrags, zu einem leeren normativen Signifikanten geworden. Leer, denn Gerechtigkeit wird in den unterschiedlichsten Diskursen als der global zu verwirklichende Standard verstanden, anhand dessen entschieden wird, was getan werden soll. Normativ, da gleichzeitig eine Vielzahl partikularer Gerechtigkeitsvorstellungen existiert, von denen sich aber eine jede als universeller Standard präsentiert. Angesichts der Vielfalt und Inkompatibilität dieser unterschiedlichen Vorstellungen erscheint eine individuelle Realisierung derselben (und zur gleichen Zeit) kaum möglich. 
Dieses Scheitern liegt nicht zuletzt darin begründet, dass Gerechtigkeit als positive Umkehrung einer negativen Situation entsteht.

Justice is an empty term and not a formal conceptual determination because it is the mere positive reverse of a situation lived originally in negative terms: deprivation, dislocation, disorder etc. This is what creates the distance between what is and what ought to be, which is the root of any ethical experience and reflection (Laclau 2004, S. 286).

Die semantische Funktion des Begriffs Gerechtigkeit besteht demnach darin, auf eine abwesende Fülle, auf einen leeren Platz im Diskurs zu verweisen, der nicht mit einer spezifischen Definition des Begriffs Gerechtigkeit geschlossen werden kann (Derrida 1991, S. 35). Darin liegt das „Universelle“ des Begriffs und auch seine Fähigkeit als fundamentale Rechtfertigung zu dienen: Nicht der Inhalt von Gerechtigkeit ist es, der all die unterschiedlichen Ansprüche eint, sondern vielmehr die Widerborstigkeit des Begriffs, sich auf einen Nenner bringen zu lassen wodurch seine beständige Anrufung im Diskurs als Horizont jedweder Forderungen nach Gerechtigkeit erst möglich wird.

Wenn Gerechtigkeit als leerer normativer Signifikant fungiert, sie also durch ihre Absenz und Unmöglichkeit präsent ist, dann ist der „ethical moment“ der Erfahrung von Gerechtigkeit die Erfahrung eines Mangels an Gerechtigkeit. In eben diesem ethischen Moment wird Gerechtigkeit durch die Distanz zwischen dem, was ist, und dem, was sein soll, konstituiert. Dies kann jedoch nicht über eine positive moralische Evaluation erfolgen, denn dies hieße „attributing to a particular content the role of bearer of one of the name of fullness" (Laclau 2004, S. 287). Aus diesem Grund kann auch die (positive) Erfahrung von Gerechtigkeit kein sicherer Grund für unser Verhalten und/oder für gerechte Ordnungen sein. „Die Evidenz, dass hier und jetzt Gerechtigkeit herrscht, sagt uns nicht, wie wir Gerechtigkeit dort und dann wiederholen können“ (Menke 2002, S. 258).

Neben dieser Erfahrung des Mangels steht aber noch mehr auf dem Spiel, denn es geht erneut um die Untrennbarkeit von Normativem, Ethischem und Politischem. Ähnlich der Demokratie ist der Gerechtigkeit eine „selbstdekonstruktive Kraft“ (Derrida 2002, S. 56) ${ }^{11}$ eingeschrieben, denn: „Wir müssen dabei auch wissen, dass sich diese Gerechtigkeit immer an das vielfältig Besondere [singularités] richtet, an die Besonderheit des anderen, unbeschadet oder gerade aufgrund ihres Anspruchs auf Universalität“ (Derrida 1991, S. 41, vgl. auch 34-35). Gerechtigkeit verlangt also einerseits die Beachtung und Geltung des Partikularen, des Besonderen, die sich aber andererseits aus dem Anspruch des Universellen, des Allgemeinen speist. Dieses Spannungsverhältnis, das dazu zwingt, das Partikulare im Universellen zu sehen, begründet zweierlei: Zum einen, dass die Gerechtigkeit „im Kommen“ (Derrida 1991, S. 56, Hervorhebung im Original) bleibt, denn eine Auflösung des Spannungsverhältnisses zu Gunsten des einen oder des Anderen ist nicht möglich - so wie eben die Realisierung aller partikularen Gerechtigkeitsvorstellungen oder die Verallgemeinerung des einen Besonderen nicht denkbar sind.

11 Ich danke einem anonymen Gutachter/einer anonymen Gutachterin für diesen Hinweis. 
Zum anderen ist die Gerechtigkeit nicht nur die Erfahrung eines ethischen Moments, sondern auch eines politischen. „In dem Maße, in dem sie nicht einfach ein [...] politischer Begriff ist, schafft darum vielleicht die Gerechtigkeit zukünftig Offenheit für eine Verwandlung, eine Umgestaltung oder eine Neu(be)gründung [...] der Politik [...].“(Derrida 1991, S. 56, Hervorhebung im Original). „Politisch, denn die Gerechtigkeit ermöglicht - zumindest potentiell - aufgrund ihrer Zukünftigkeit die Aufbrechung herrschender [...] Verhältnisse “ (Flügel-Martinsen 2009, S. 193), sie erlaubt also die Kritik bestehender hegemonialer Strukturen und befeuert so den ethischen und den politischen Deutungsstreit über die Gerechtigkeit - wie das Illustrationsbeispiel dieses Beitrags zeigt.

Wenn Gerechtigkeit nun als leerer normativer Signifikant fungiert, wenn sie immer im Kommen ist, wie kann sie dann trotzdem im Hier und Jetzt möglich werden? Im Sinne des Arguments des Beitrags geht es hier um die gerechte Praxis. Weil die Erfahrung von Gerechtigkeit Kraft und Grund in einem ist („als Kraft treibt sie uns an und immer weiter; als Grund ist sie gerichtet auf eine Ordnung, die sie einsetzt" [Menke 2002, S. 255]), bedarf es einer Diskurspraxis des Entscheidens, die sich über die Unmöglichkeit und Zukünftigkeit von Gerechtigkeit im Klaren ist. Eben das sind die Bedingungen der gerechten Praxis, die ein bestimmtes Verhalten - „primär: das der Entscheidung“ (Menke 2002, S. 247) - erfüllen muss, um als gerecht bezeichnet zu werden. Um gerecht sein zu können, deutet und stiftet die Entscheidung die Gerechtigkeit (Derrida 1991, S. 47). Doch die Entscheidung fällt in einem Terrain der Unentscheidbarkeit, in einem Feld der Unmöglichkeit, Entscheidungen letztgültig zu fixieren. Möglich sind (wie oben erläutert) partielle Fixierungen, von dem, was gerecht ist. Insofern ist Festschreibung der „letztgültigen“ Bedeutung - hier die der Gerechtigkeit - immer aufgeschoben; es gibt keinen Moment, in dem Bedeutung vollständig erreicht werden könnte, ist Gerechtigkeit doch stets im Kommen. Allerdings ist jede Festschreibung der Gerechtigkeit in einem Diskurs eine Schließung dieses Diskurses (Laclau u. Mouffe 2001, S. 111-112), denn in einem Terrain der Unentscheidbarkeit suchen Subjekte nach einer konstitutiven Entscheidung, um gerechtes Verhalten eher in der einen denn in der anderen Weise zu artikulieren bzw. zu begründen, also das Partikulare zum Universellen zu machen und Gerechtigkeit nicht mehr im Kommen zu verstehen. Mit Blick auf das dominante Verständnis von Drogen im globalen Diskurs bedeutet das: Drogen sind illegal, ihr Gebrauch ist abweichendes Verhalten, daher ist eine Liberalisierung der globalen Drogenpolitik der falsche Weg; vielmehr gilt es Gerechtigkeit über die Bekämpfung der sozialen, gesellschaftlichen Ursachen von Drogenkonsum herzustellen.

Nur, diese Suche nach einer Begründung für die Gerechtigkeit einer Entscheidung - gegen Liberalisierung von Drogen, für gerechte soziale Reformen -, „, dass also die Gerechtigkeit einer Entscheidung etwas ist, das wir durch die Weise unserer Verhaltens sicherstellen können“ (Menke 2002, S. 254), funktioniert nicht, weil „die Gerechtigkeit einer Entscheidung durch ihre guten Gründe nicht sichergestellt werden kann, macht ein Entscheiden, eine Weise des Entscheidens, die sich eben das zutraut, Gerechtigkeit gerade unmöglich“ (Menke 2002, S. 254). Insofern ist eine gerechte Praxis eine, die um die Unmöglichkeit und Zukünftigkeit 
von Gerechtigkeit weiß, die aber den Glauben an diese Praxis verteidigt und zugleich preisgibt - darin besteht die Aporie (Menke 2002, S. 255).

Zieht man nun das oben formulierte Verständnis poststrukturalistischer IBAutor_innen von Ethik als relational, als einer Bindung an und Verantwortung für den Anderen heran, so verweist die gerechte Praxis nicht nur auf die Unmöglichkeit von Gerechtigkeit, sondern auch darauf, dass eine gerechte Praxis über den relationalen Charakter von Gerechtigkeit reflektiert: Während ein Individuum durch die Erfahrung von Ungerechtigkeit für sich festlegen kann, was er/sie für gerecht oder ungerecht hält, so wird diese Ungerechtigkeit dadurch nicht geheilt - vielmehr erfolgt dies über die Verbindung zum Anderen, über die Verbindung zu der vom Anderen erfahrenen Ungerechtigkeit (Derrida 1991, S. 41). Verknappt gesagt: Meine Gerechtigkeit kann nicht ohne die meines Anderen realisiert werden. Zugegeben, es handelt sich hier um eine äußerst diffizile Situation, aus der es keinen direkten Ausweg gibt. Die Entscheidung verlangt daher einen Vertrauensvorschuss (, a leap of faith “ [Derrida 2003, S. 118]), d. h. sich der Schwierigkeit der Entscheidung bewusst zu sein und trotzdem weiter nach Gerechtigkeit zu streben - die oben angesprochene Aporie. Letztlich spiegelt sich in einer solchen Konzeption von gerechter Praxis die Frage, in welcher Verbindung wir zum Anderen stehen. Liegt in dem Versuch der Fixierung einer bestimmten Bedeutung von Gerechtigkeit doch nicht nur die Gefahr mehr Ungerechtigkeit zu produzieren, sondern auch die Relation zum Anderen zu unterminieren und seiner Verantwortung für den Anderen nicht gerecht zu werden. Ein Punkt, der im globalen Drogendiskurs vor allem mit Blick darauf thematisiert wird, dass der „Krieg gegen die Drogen“ nicht nur die Drogenhändler_innen trifft, sondern eben auch eine Vielzahl an Anderen wie Abhängige und deren Angehörige oder gänzlich unbeteiligte Dritte.

Eine gerechte Praxis kann aufgrund dieser „eingebauten“ Relationalität auch die Gefahr ausräumen, dass wir in dem Verweis auf unsere Verantwortung für den Anderen allzu leicht die Beziehung vergessen, in der wir längst zu dem Anderen stehen - also weniger über das Sein, sondern mehr über das Sollen nachdenken (Zehfuss 2007, S. 70). Die Beziehung zum Anderen kann nicht machtfrei gedacht werden - ein Umstand, der sich darin widerspiegelt, dass die VN erstmals 1983 (und damit mehr als 20 Jahre nach Entstehung des Drogenprohibitionsregimes) Nichtregierungsorganisationen (NROs), zivilgesellschaftliche Gruppen und damit Kritiker_innen der VN-Drogenpolitik institutionell in die Diskussion um Prohibition einband. Diese Einbindung begann sich jedoch erst 15 Jahre später, im Rahmen der Sondertagung der VN-Generalversammlung über Drogen (UNGASS), in kleinem Maße auszuzahlen. ${ }^{12} 2008$ stellte das globale Forum Beyond 2008 dann erstmals eine umfassende Plattform für die Zivilgesellschaft dar,

12 Obwohl das Vienna NGO Committee on Narcotic Drugs bereits seit 1983 existiert und als institutionelle Verbindung zwischen dem Büro der VN für Drogen- und Verbrechensbekämpfung (UNODC), der VN-Suchtstoffkommission (CND) und NROs fungiert, war der Einfluss von NROs vor 1998 kaum fühlbar. Zudem haben sich die meisten NROs erst um und nach der UNGASS von 1998 gegründet. 
sich in die Überprüfung der Ziele der 1998er UNGASS einzubringen. ${ }^{13}$ Die Einbindung bescherte diesen Gruppen einerseits eine institutionalisierte Funktion, andererseits ist ihre Rolle beratend und vor allem dann erschwert, wenn sich ihre Empfehlungen gegen die restriktive Drogenpolitik des Büros der VN für Drogenund Verbrechensbekämpfung (UNODC) richten. Der über Jahrzehnte hinweg geschlossene Drogendiskurs musste sich zumindest der Einbindung von kritischen Akteuren_innen im Rahmen der Forderung einer „gerechten und wirksamen Drogenpolitik“14 einen Spaltbreit öffnen.

Darin zeigt sich, dass eine gerechte Praxis keine Aufgabenteilung zwischen dem Ethischen und dem Politischen vornimmt, sondern die kritische Reflexion über Ausschluss und Marginalisierung verweist auf einen inklusiven, offenen Austausch über Gerechtigkeitsvorstellungen in gegenseitigem agonistischem Respekt trotz differenter Auffassungen (Connolly 1995) und weniger auf einen Dialog, der primär auf Konsens zielt. Führt doch die Fragmentiertheit des Gerechtigkeitsdiskurses dazu, dass jede noch so abstrakte, generelle Definition von Gerechtigkeit Widerspruch generieren würde, und insofern meint offener Austausch im Rahmen der gerechten Praxis auch, dass die Beteiligten dieses Austauschs um die Unmöglichkeit und Zukünftigkeit von Gerechtigkeit wissen. Ein Austausch über Gerechtigkeit, der durch die Leere und Unmöglichkeit derselben generiert wird, weist einerseits daraufhin, dass der Diskurs über Gerechtigkeit nicht geschlossen werden kann, zum anderen aber auch darauf, dass dieser Diskurs auch nicht geschlossen werden soll. Denn der autologische Charakter poststrukturalistischer Ansätze erlaubt hier nicht nur eine Praxis des Entscheidens, die sich über die Unmöglichkeit von Gerechtigkeit im Klaren ist, sondern eben auch die angesprochene Kritik, die Formen der Exklusion und Hegemonie offenlegt (Glynos u. Howarth 2007, S. 192) und letztlich eine gerechte Praxis ermöglicht. Stark zugespitzt bedeutet eine gerecht Praxis demnach, den Diskurs nicht schließen zu wollen (gerade aufgrund der Erkenntnis, dass dies auch nicht möglich ist).

\section{Implikationen für globale Sicherheitspolitik: Gerechtigkeit im globalen Drogendiskurs}

In den vorherigen Abschnitten wurde bereits auf den globalen Drogendiskurs verwiesen. Dabei zeigte sich in den verschiedenen Zitaten, dass unterschiedliche Akteure Gerechtigkeit in der globalen Drogenpolitik einfordern. Es zeigte sich auch, dass sie unter Gerechtigkeit Unterschiedliches, zum Teil Konträres verstehen. So entsteht mehr Gerechtigkeit in der globalen Drogenpolitik für den Papst, wenn man die gesellschaftlichen Ursachen des Drogenkonsums angeht (siehe Eingangszitat). Es geht ihm dabei offenbar um eine Gerechtigkeit, die - aufgrund seiner AntiLiberalisierungsposition - stärker im Einklang mit dem globalen Drogenprohibiti-

13 Das Forum wurde von rund 300 NRO-Repräsentanten_innen besucht, die sich über alternative Drogenpolitiken austauschten und Empfehlungen für die künftigen Richtungen der VN-Drogenpolitik abgaben (Vienna NGO Committee on Narcotic Drugs 2008).

14 So der Titel des Manifests einer Globalkoalition von NROs anlässlich der UNGASS 1998 (European Coalition for Just and Effective Drug Policies 1998). 
onsregime steht, das eine „society free from drug abuse and [...] illicit traffic“ (United Nations 1973a, S. 8) erreichen will. In den Verhandlungen, aus denen das Regime von 1961 an entstand, wurde der Weg in diese Welt frei von Drogen klar vorgezeichnet: über ein universell akzeptiertes Verbot und eine internationale Kontrolle der Drogen (United Nations 1964b, S. 6, 1973a, S. 1). Gerechtigkeit und damit eine gerechte Drogenpolitik erwächst hier aus dem Anspruch der Staaten, ihre Bürger vor den Gefahren der Drogen zu schützen (Walker 2006). Das ist eine andere Referenz auf Gerechtigkeit als die von Perla de la Rosa, Schauspielerin aus Ciudad Juárez in Mexiko: „Die Militarisierung bringt uns nicht weiter. Sie sorgt für immer neue Gewalttaten gegen Frauen, Kinder und Jugendliche. Wir brauchen einen Dialog, eine friedliche Lösung und Gerechtigkeit in Mexiko" (Perla de la Rosa, zitiert nach Henkel 2011). Ihr geht es um soziale Gerechtigkeit, um ein Ende des „Drogenkriegs“, der kein gerechter sein kann, da er lediglich Ungerechtigkeitserfahrungen produziert. Es ist auch eine andere Referenz auf Gerechtigkeit als die von David Simon, Schöpfer der US-Fernsehserie „The Wire“, die den Zerfall der US-Großstadt Baltimore durch den „Drogenkrieg“ auf den unterschiedlichsten gesellschaftlichen Ebenen (Drogenhändler_innen, Polizisten_innen, Journalisten_innen, Lehrer_innen, Schüler_innen) zeigte. ${ }^{15}$ Der „Krieg gegen die Drogen“ werde heute nur noch als ein „gerechter Krieg“ wahrgenommen, weil die Amerikaner glauben, „dass es wirklich gegen Drogen geht - und nicht gegen die Unterschicht“, denn diese sei das eigentliche Ziel. ${ }^{16}$ Hier ist es der Mangel an Erfahrung von Ungerechtigkeit, der zur Wahrnehmung von Gerechtigkeit führt.

Diese zu Illustrationszwecken ausgewählten unterschiedlichen Zitate, von unterschiedlichen Akteuren auf unterschiedlichen gesellschaftlichen und politischen Ebenen, verweisen auf die Konstruktion von Gerechtigkeit als normativen leeren Signifikanten im globalen Drogendiskurs. Einerseits ist Gerechtigkeit durch ihren Mangel präsent. Darin liegt, wie oben ausgeführt, der ethische Moment, in dem sich Gerechtigkeit durch die Distanz zwischen dem, was ist, und dem, was sein soll, konstituiert. Die Referenz auf den Mangel von Gerechtigkeit von Perla de la Rosa verweist darauf, aber auch die des Papstes, verlangen doch beide, dass jetzt und hier Gerechtigkeit geschehen soll, da jetzt und hier Ungerechtigkeit herrscht. Und auch, das wurde ebenfalls oben erläutert, dass die positive Erfahrung von Gerechtigkeit hier und jetzt kein sicherer Grund für unser Verhalten und/oder für gerechte Ordnungen sein kann, denn solange der amerikanischen Unterschicht laut Serienautor Simon suggeriert wird, der „Krieg gegen die Drogen“ sei ein gerechter, sei deren Erfahrung von Gerechtigkeit eine Chimäre, die die Sicht auf die bestehenden Machtverhältnisse verstellt.

Nun wurde in den theoretischen Passagen des Beitrags ebenfalls bereits betont, dass Gerechtigkeit aus poststrukturalistischer Sicht als leerer normativer Signifikant mit universellem Anspruch auftritt und eine diskursive Schließung anstrebt. Diese Schließung entsteht durch die Strukturierung von Praktiken in einem Terrain der Unentscheidbarkeit: Politische Ordnungen beinhalten/bedeuten die Ex-

15 http://www.hbo.com/the-wire/index.html. Zugegriffen: 08.01.2015.

16 Interview mit David Simon unter http://www.brandeins.de/archiv/2012/kapitalismus/so-schafftman-soziopathen.html. Zugegriffen: 08.02.2015. 
klusion von bestimmten Möglichkeiten und damit auch den Versuch, ein diskursives Feld zu schließen. Ob dieser Versuch der Schließung einen antagonistischen Charakter hat, also ob die Elemente, die ausgeschlossen werden, als Gefahr und Bedrohung angesehen werden, hängt von dem mehr oder weniger hegemonialen Charakter der Praktiken ab, d. h. davon, wie sehr sie eine Forderung mit universellem Anspruch artikulieren und Gerechtigkeit als nicht mehr beständig im Kommen betrachten. Der Begriff Hegemonie meint dabei, dass bestimmte Formationen diskursiven Sinns (eine spezifische Deutung/Interpretation eines Phänomens wie bspw. Drogen als Gefahr für die Menschheit) zu machtvollen, dominanten Formationen werden. Letztere setzen sich durch, indem sie als „normal“, „richtig“, „sinnvoll“ erscheinen. Diese Durchsetzung hat entscheidend damit zu tun, dass die Formationen beanspruchen, ein soziales Projekt - eine „Welt frei von Drogen“ - in seiner Gesamtheit einheitlich zu repräsentieren. Diese „Einheitsrepräsentation“ funktioniert jedoch durch Grenzziehungen, zeichnet sich jede Hegemonie doch zwangsläufig durch Einschluss und Ausschluss aus, denn die Gesamtheit wird erst sichtbar durch die Abgrenzung von anderen Formationen oder Projekten. Hegemoniale Praktiken versuchen insofern, einen Diskurs zu schließen, um einen vollständig „genähten“ Raum von Repräsentation zu konstituieren (Torfing 1999, S. 101; vgl. auch Nonhoff 2006).

Der globale Drogendiskurs ist voll von solchen versuchten hegemonialen Schließungen. Dies zeigt sich besonders eindrücklich mit Blick auf das globale Drogenprohibitionsregime und sein Verständnis von Drogen. Heute sprechen wir von Drogen als Problem - ein Problem, das sie wurden: Die heutige Sicht ist das Ergebnis einer seit dem frühen 20. Jahrhundert laufenden politischen Auseinandersetzung, durch die sich das heutige Verständnis von Drogen als illegal und ihr Konsum als abweichendes Verhalten durchgesetzt hat. Diese Durchsetzung einer partikularen Vorstellung gelang vor allem durch die Institutionalisierung dieses spezifischen Verständnisses: Mit drei VN-Konventionen seit 1961 entstand über die Jahrzehnte ein globales Drogenprohibitionsregime (Nadelmann 1990), das von nahezu allen Staaten der Welt unterzeichnet wurde. Seine weitreichenden Restriktionen für Staaten in Bezug auf die Produktion und den Verkauf von Drogen, aber auch die Kriminalisierung des Konsums und Besitzes von Drogen, werden als gerechtfertigt und richtig empfunden, um einen globalen „drug holocaust“ (United Nations 1991, S. 9) zu verhindern und die Welt vom Übel der Drogen zu befreien.

So hat sich das Prohibitionsregime mit seinem hegemonialen Verständnis von Drogen als „evil“ (United Nations 1964a, S. 6) über die Jahrzehnte hinweg zu einem normativen Standard für erwünschtes Verhalten entwickelt. Der Moment der Schließungen ist insofern entscheidend für hegemoniale Ordnungen wie das Drogenregime (Stäheli 1999, S. 149-150; Laclau 2000b, S. 304). Diese erfolgen über den Ausschluss dessen, was der Diskurs nicht einschließt, Elemente des Anderen: Unordnung, Entbehrung, Ungerechtigkeit. In Sicherheitsdiskursen wie dem Drogendiskurs sind Differenz und Ausschluss besonders weitreichend: Werden doch nicht nur Drogen als das böse Andere ausgeschlossen, sondern auch alles, was sich als ihnen ,zugehörig“ konstruieren lässt: vom internationalen Drogenhandel, der internationalen Drogenmafia, dem/der international agierenden Drogenhändler_in (,the kingpins of the international traffic“ [United Nations 1964b, S. 121]) über 
den/die Drogendealer_in auf der Straße bis hin zu Drogensüchtigen („liars and active proselytizers" [United Nations 1973b, S. 183]) und dem/der, der/die Drogen anbaut (für eine ausführliche Analyse siehe Herschinger 2011).

Auf der Basis dieser Äquivalenzierung von ausgeschlossenen Anderen rechtfertigt das Prohibitionsregime bspw. die Darstellung von Drogenabhängigen wahlweise als Kriminelle, als medizinisches oder psychologisches Problem oder als Opfer der Umstände (United Nations 1964a, S. 107, 111, 113, 1991, S. 6, 17). Gemäß der ersten Sicht sind Abhängige ebenso „böse“ wie Drogenhändler_innen oder -dealer_innen, die die Fundamente der Gemeinschaft der Nicht-Süchtigen gefährden: Abhängigkeit ist ein „morbid habit“ (United Nations 1964a, S. 60), dessen Verbreitung Süchtige anstreben und das als ,anti-social behavior which was to threat the whole community “ (United Nations 1973b, S. 183) qualifiziert werden muss; letztlich sind sie kriminelle Psychopaten. Den Süchtigen als Opfer und die Abhängigkeit als ein medizinisches oder psychologisches Problem zu beschreiben, bedeutet wiederum, in diesen Opfern kein Gefahrenpotenzial zu sehen. Als Opfer der Umstände haben Süchtige ein Recht auf Rehabilitation - das schuldet ihnen die Gemeinschaft, denn schließlich werden sie so wieder „useful citizen[s]“ (United Nations 1964a, S. 105, 1991, S. 9, 12-13, 1973b, S. 53, 1973a), in der Lage, ein normales Leben zu führen. Hier ist die Abhängigkeit der Schuldige: Isoliere die Person von ihrer Abhängigkeit und sie kann zurückkehren in die Gemeinschaft. Diese „gesundheitsorientierte/medizinische“ Interpretation soll keinesfalls darüber hinwegtäuschen, dass sie sich mit der Kriminalisierung trifft: Solange ein Mensch Drogen nimmt, solange ist er Teil des feindlichen Anderen, genauso wie Drogenbarone_innen, Dealer_innen und die Drogen selbst. Diese Fixierungen ergeben das gleiche Resultat: Abhängige sind Andere, Ausgeschlossene.

An der versuchten Fixierung der Bedeutung von Drogenabhängigkeit lässt sich die Kontingenz und Temporalität ersehen, von denen Diskurse durchzogen sind. Die ultimative Festschreibung der Kriminalität von Drogenabhängigen ist unmöglich, sie hielt sich aber über einen gewissen Zeitraum. Spätestens jedoch durch den Auftritt von NROs und anderen zivilgesellschaftlichen Gruppen geriet sie unter Druck. So, wenn der Papst davon spricht, dass man die hinter der Sucht liegenden Probleme angehen müsse, „indem man sich für mehr Gerechtigkeit einsetzt“; so, wenn deutlich gemacht wird, dass es sich bei Abhängigen viel zu oft um benachteiligte Gruppen („,black, ethnic minorities or indigenous peoples“ [Transnational Institute 2008, S. 36]) handle, deren Stigmatisierung dadurch noch verschärft werde; so aber auch, wenn einige europäische Regierungen versuchen, die Frage von Abhängigkeit nicht mehr auf globaler Ebene zu lösen, sondern auf nationaler (bspw. mit sogenannten Fixerstuben) (Drogenbeauftragte der Bundesregierung 2003, S. 27-43). Insofern eröffnen die Fixierungen durch das globale Prohibitionsregime Raum für Kritik und Befragung ihrer normativen Positionen (Glynos u. Howarth 2007, S. 197), die bis heute von den orthodoxen Interpreten des Regimes zurückgewiesen wird, weil gerade Fixerstuben oder Substitutionsprogramme immoralische Aktivitäten für einen Staat seien, da sie den Konsum und den Handel von Drogen fördern und damit schwerwiegende normative und politische Probleme produzieren würden (Mission interministérielle de la lutte contre la drogue et la toxicomanie 1999, S. 110-111; vgl. auch Bewley-Taylor 2012). 
Wenn nun Gerechtigkeit im Sinne einer gerechten Praxis bedeutet, einen Diskurs nicht schließen zu wollen, dann stehen derartige hegemoniale Strukturierungen, die auf Schließungen eines Diskurses beruhen, der Gerechtigkeit entgegen. Präziser gesagt: Im globalen Drogendiskurs stellt sich das Prohibitionsregime als eine Schließung dar, die ein bestimmtes Verständnis von Gerechtigkeit und der darauf basierenden Begründungen für bestimmte Politiken - wie mit Drogenabhängigkeit umzugehen ist - festzuschreiben sucht. Zugespitzt: Hegemoniale Ordnungen machen aufgrund des permanenten Versuchs der Schließung, d. h. der Festschreibung von dem, was Gerechtigkeit ist, Gerechtigkeit unmöglich - sie ist dann schlicht nicht mehr im Kommen. Und da hegemoniale Ordnungen Widerstand auszuschließen suchen, stehen sie sowohl einer Praxis der Kritik und Befragung als auch einer gerechten Praxis im Wege. Ihre Praktiken sind keine gerechte Praxis, denn diese würde über die Unmöglichkeit und Zukünftigkeit von Gerechtigkeit reflektieren, sie aber ohne den Versuch der Schließung des Diskurses trotzdem anstreben.

Letzteres wäre denkbar, wenn reflektiert würde, was genau diese hegemonialen Praktiken verdunkeln. Es ist die oben angesprochene Relation, die Bindung des Selbst an und seine normative Verantwortung für den Anderen, die dadurch verschleiert wird. Im Falle des Drogendiskurses ist es zuallererst die Bindung an die Drogenabhängigen. Die dargestellten drei „Varianten“ der Konstruktion des Drogenabhängigen werden mit Blick auf die Norm des Drogenregimes („Drogen sind illegal und ihr Konsum abweichendes Verhalten“) gerechtfertigt. Durch diese Konstruktionen wird eine exklusive und restriktive Sicht auf Subjektivität begründet, die nur denen Subjektqualität zugesteht, die sich nicht durch Abhängigkeiten korrumpieren haben lassen und alle Anderen - hier die Abhängigen - zu Objekten von politischen, rechtlichen, aber eben auch kriegerischen Aktivitäten macht. „We regard people as sovereign over their bodies and - besides the right this implies regarding what adults can put into their bodies and how - INPUD also recognises the individual's right to freely interpret their experiences of drug-taking using a disease or any other model“ (International Network of People Who Use Drugs o. D.). Solche Ansichten haben einen schweren Stand, da ihre Sicht auf Drogen nicht mit der hegemonial institutionalisierten Norm des Regimes übereinstimmt und insofern weder als gerechtfertigt noch als gerecht angesehen wird.

Diese restriktive Sicht auf Subjektivität ist problematisch für die Realisierung der normativen Verantwortung. Indem Exklusion hier über die Konstruktion von „independent sites of evil“ erfolgt, sprich über "those differences that pose the greatest threat to the integrity and certainty " (Connolly 1991, S. ix) für eine Gemeinschaft wie die der Nicht-Süchtigen, setzt diese in problematischer und kontingenter Form die Grenzen von Verantwortung. So wird verhindert, zu erkennen, dass wir in Beziehungen zu anderen stehen, dass das Selbst in ein unauflösliches Netzwerk von Anderen eingebunden ist (Hansen 2006, S. 40). Letztlich wird damit die Relationaliät der Beziehung verdunkelt und die politische Dimension von der ethischen getrennt. Insofern: „, [T] hings have already gone wrong“ (Zehfuss 2013, S. 162), was das Drogenregime betrifft. Dessen Praktiken verhindern also nicht nur, die Verantwortung für den Anderen zu erkennen, sondern sie vermindern auch das Maß, in dem Subjekte sich über die radikale Kontingenz ihrer Pra- 
xis im Klaren sind oder sein können. Daraus erwächst keine gerechte Praxis. Steht diese doch letztlich für eine inklusive Sicht auf Subjektivität.

\section{Fazit}

Dieser Beitrag hat sich zum Ziel gesetzt, aufzuzeigen, wie Gerechtigkeit in den IB poststrukturalistisch gelesen werden kann Dazu argumentierte er, dass Gerechtigkeit ein leerer normativer Signifikant ist. Da die Erfahrung von Gerechtigkeit die Erfahrung eines Mangels ist, wird sie durch die Distanz zwischen dem, was ist, und dem, was sein soll, konstituiert. Aufgrund dieser Distanz, vor allem aber da Gerechtigkeit stets im Kommen ist, bedarf es einer Diskurspraxis des Entscheidens, die sich über die Unmöglichkeit und Zukünftigkeit von Gerechtigkeit im Klaren ist. Eine solche Praxis lässt sich als gerechte Praxis beschreiben, da sie Gerechtigkeit anstrebt, obwohl ihr die Unmöglichkeit und beständige Zukünftigkeit derselben klar ist und sie um ihr Scheitern im Vorhinein weiß. Die gerechte Praxis verweist zum einen darauf, dass der Diskurs über Gerechtigkeit nicht geschlossen werden kann. Zum anderen macht sie klar, dass dieser Diskurs auch nicht geschlossen werden soll. Insofern bedeutet Gerechtigkeit, den Diskurs nicht schließen zu wollen. Eine solche Lesart von Gerechtigkeit, so das Argument, ist produktiv für eine alternative und kritische Analyse von internationaler Politik die „Alltagsweisheit“ eines normativen Defizits poststrukturalistischer Ansätze widerlegt sie. Der Beitrag verweist so darauf, dass normative Fragen auf unterschiedlichste Weisen zum Zentrum der Betrachtungen gemacht werden können und dass es in den IB - und damit auch in dem neuen Feld der Internationalen Politischen Theorie - nicht nur eine privilegierte Vorstellung davon gibt (und geben sollte), wie normative Fragen erörtert werden können. Mag die Umstrittenheit von Gerechtigkeit zu unangenehm vielen Verständnissen derselben führen, die Umstrittenheit der Zugänge zu normativen Fragen und die Vielfalt von Normativitätsverständnissen in den IB sind dagegen begrüßenswert.

Die Illustration anhand des globalen Drogendiskurses zeigt, dass gerade hegemoniale Ordnungen aufgrund des permanenten Versuchs der Schließung, d. h. der Festschreibung von dem, was Gerechtigkeit ist, letztere unmöglich machen. Was sie allerdings ermöglichen können, ist eine gerechte Praxis - indem die ethische nicht von der politischen Dimension getrennt wird und mit der Aporie, das Gerechtigkeit zwar unmöglich und stets im Kommen ist, aber nichtsdestotrotz angestrebt werden soll, umgegangen wird. Gerade im Drogendiskurs im Zeitalter des „Kriegs gegen die Drogen“ könnte dies umgesetzt werden - nicht nur, indem die Militarisierung ein Ende findet (denn hier wird ja gerade die ethische Frage von der politischen unter dem Vorwand einer „politics of protection“ gelöst [Walker 2006]). Sondern auch, indem die Frage, in welcher Verbindung wir zum Anderen stehen, thematisiert und damit auch anerkannt wird, dass Nachfrage und Angebot von Drogen eng miteinander verknüpft sind; dass die Verantwortung für die eigenen Bürger_innen gleich ob Konsumenten_innen oder nicht - nicht nur heißt, keinen Krieg gegen sie zu führen, sondern ebenso, dass die Verantwortung für die Etablierung von Gerechtigkeit hier und jetzt auch für die Anderen dort und dann heißt - und nicht die Gefahr von mehr Ungerechtigkeit bergen sollte. Diese inklusive Sicht auf Subjektivität 
bedeutet, dass Verantwortung gilt, gleich, ob es sich um Nicht-Konsumenten_innen, Drogenabhängige oder Bauern/Bäuerinnen in Produktionsländern handelt. Denn solange die Erfahrung von Ungerechtigkeit nicht nur von denen kommt, die den meisten Profit aus Drogen ziehen, sondern ebenso durch das globale Drogenprohibitionsregime und den „Krieg gegen die Drogen“, wird sich der Ruf nach Gerechtigkeit vor allem gerade an jene richten, die aufgrund ihres demokratischen Ohrs dafür offen und nicht durch hegemoniale Schließungen ihrer politischen Ordnungen taub sein sollten - sprich an jene, die das Regime geschaffen haben. Aus diesem Grund ist eine gerechte Praxis entscheidend für das (wenngleich zum Scheitern verurteilte) Streben nach der Gerechtigkeit, das sich den Versuchen, den Diskurs schließen zu wollen - um der Gerechtigkeit willen - entgegenstellt.

\section{Literatur}

Arneson, Richard J. 2006. Justice after Rawls. In The Oxford Handbook of Political Theory, Hrsg. John S. Dryzek, Bonnie Honig und Anne Phillips, 45-64. Oxford: Oxford University Press.

Ashley, Richard K., und R.B.J. Walker. 1990. Reading Dissidence/Writing the Discipline: Crisis and the Question of Sovereignty in International Studies. International Studies Quarterly 34: 367-416.

Bell, Duncan (Hrsg.). 2010. Ehics and World Politics. Oxford: Oxford University Press.

Bewley-Taylor, David. 2012. International Drug Control: Consensus Fractured. Cambridge: Cambridge University Press.

Bonacker, Thorsten. 2002. Die Gemeinschaft der Dekonstruktion: zum normativen Gehalt liberaler Gemeinschaften. In Philosophie der Dekonstruktion. Zum Verhältnis von Normativität und Praxis, Hrsg. Andrea Kern und Christoph Menke, 264-288. Berlin: Suhrkamp Verlag.

Brown, Chris. 1992. International Relations Theory. New Normative Approaches. New York: Columbia University Press.

Brown, Chris. 1994. 'Turtles All the Way Down': Anti-Foundationalism, Critical Theory and International Relations. Millennium: Journal of International Studies 23: 213-236. DOI: $10.1177 / 03058298940230020901$.

Bulley, Dan. 2006. Negotiating ethics: Campbell, ontopology and hospitality. Review of International Studies 32: 645-663. DOI: 10.1017/S0260210506007200.

Burke, Anthony. 2013. Security as Ethics. In The Routledge Handbook of New Security Studies, Hrsg. Peter Burgess, 90-99. London/New York, NY: Routledge.

Campbell, David. 1993. Politics Without Principle. Sovereignty, Ethics, and the Narratives of the Gulf War. Boulder: Lynne Rienner.

Campbell, David. 1998a. National Deconstruction. Violence, Identity, and Justice in Bosnia. London/Minneapolis: University of Minnesota Press.

Campbell, David. 1998b. Writing Security. United States Foreign Policy and the Politics of Identity (revised edition). Minneapolis: University of Minnesota Press.

Campbell, David. 1999. The Deterritorialization of Responsibility: Levinas, Derrida, and Ethics after the End of Philosophy. In Moral Spaces. Rethinking Ethics and World Politics, Hrsg. David Campbell und Michael J. Shapiro, 29-55. Minneapolis: University of Minnesota Press. 
Campbell, David, und Michael J. Shapiro. 1999a. Introduction. From Ethical Theory to the Ethical Relation. In dies.: Moral Spaces. Rethinking Ethics and World Politics, VII-XX. Minneapolis: University of Minnesota Press.

Campbell, David, und Michael J. Shapiro (Hrsg.). 1999b. Moral Spaces. Rethinking Ethics and World Politics. Minneapolis: University of Minnesota Press.

Campbell, Elaine. 2011. The cultural politics of justice: Bakhtin, stand-up comedy and post-9/11 securitization. Theoretical Criminology 15: 159-177. DOI: $10.1177 / 1362480610387967$.

Chwaszcza, Christine. 2011. Moral Responsibility and Global Justice. A Human Rights Approach. Baden-Baden: Nomos Verlag.

Cochran, Molly. 1995. Postmodernism, Ethics and International Political Theory. Review of International Studies 21: 237-250.

Connolly, William E. 1991. Identity/Difference. Democratic Negotiations of Political Paradox. Ithaca, London: Cornell University Press.

Connolly, William E. 1995. The Ethos of Pluralization. Minneapolis, MN: University of Minnesota Press.

Couzens Hoy, David. 2004. Critical Resistance. From Poststructuralism to Post-Critique. Cambridge, MA: MIT press.

Critchley, Simon. 1992. The Ethics of Deconstruction. Derrida and Levinas. Edinburgh: Edinburgh University Press.

Deitelhoff, Nicole. 2010. Parallele Universen oder Verschmelzung der Horizonte? Zeitschrift für Internationale Beziehungen 17: 279-292.

Der Derian, James, und Michael J. Shapiro (Hrsg.). 1989. International/Intertextual Relations. Postmodern Readings of World Politics. New York: Lexington Books.

Derrida, Jacques. 1976. Die Schrift und die Differenz. Frankfurt a. M.: Suhrkamp Verlag.

Derrida, Jacques. 1991. Gesetzeskraft. Der „mystische Grund der Autorität“. Frankfurt a. M.: Suhrkamp Verlag.

Derrida, Jacques. 2002. Politik der Freundschaft. Frankfurt a. M.: Suhrkamp Verlag.

Derrida, Jacques. 2003. Autoimmunity: Real and Symbolic Suicides. In Philosophy in a Time of Terror. Dialogues with Jürgen Habermas and Jacques Derrida, Hrsg. Giovanna Borradori, 85-136. Chigaco, IL.: The University of Chicago Press.

Diez, Thomas. 1999. Die EU Lesen. Diskursive Knotenpunkte in der Britischen Europadebatte. Opladen: Leske + Budrich.

Dillon, Michael. 1999. Another Justice. Political Theory 27: 155-175.

Dillon, Michael. 2013. Deconstructing International Politics. Abingdon, New York: Routledge.

Drogenbeauftragte der Bundesregierung. 2003. Aktionsplan Drogen und Sucht. http:// www.drogenbeauftragte.de/fileadmin/dateien-dba/Service/Publikationen/Drogen_und_ Suchtbericht_2003_Drogenbeauftragte.pdf. Zugegriffen: 08.01.2015.

Dryzek, John S., Bonnie Honig, und Anne Philips (Hrsg.). 2006. The Oxford Handbook of Political Theory. Oxford: Oxford University Press.

Edkins, Jenny. 1999. Poststructuralism \& International Relations. Bringing the Political Back in. Boulder, CO/London: Lynne Rienner.

Engelkamp, Stephan, Katharina Glaab, und Judith Renner. 2012. „In der Sprechstunde“: Wie (kritische) Normenforschung ihre Stimme wiederfinden kann. Zeitschrift für Internationale Beziehungen 19: 101-129. 
European Coalition for Just and Effective Drug Policies. 1998. Für eine gerechte und wirksame Drogenpolitik. http://www.encod.de/info/FUR-EINE-GERECHTE-UNDWIRKSAME.html. Zugegriffen: 08.01.2015.

Fagan, Madeleine. 2013. Ethics and Politics after Poststructuralism: Levinas, Derrida and Nancy. Edinburgh: Edinburgh University Press.

Flügel-Martinsen, Oliver. 2009. Entzweiung. Die Normativität der Moderne. Baden-Baden: Nomos Verlag.

Flügel-Martinsen, Oliver. 2009. Grundfragen politischer Philosophie. Eine Untersuchung der Diskurse über das Politische. Baden-Baden: Nomos Verlag.

Flügel-Martinsen, Oliver. 2010. Die Normativität von Kritik. Ein Minimalmodell. Zeitschrift für Politische Theorie 1: 139-154.

Forst, Rainer. 2004. Moralische Autonomie und Autonomie der Moral. Zu einer Theorie der Normativität nach Kant. Deutsche Zeitschrift für Philosophie 52: 179-197.

Forst, Rainer. 2007. Das Recht auf Rechtfertigung. Frankfurt a. M.: Suhrkamp Verlag.

Forst, Rainer, und Klaus Günther. 2011. Die Herausbildung normativer Ordnungen. Zur Idee eines interdisziplinären Forschungsprogramms. In dies.: Die Herausbildung normativer Ordnungen. Interdisziplinäre Perspektiven, 11-30. Frankfurt a. M.: Campus Verlag.

Frost, Mervyn. 1998. A turn not taken: Ethics in IR at the Millenium. Review of International Studies 24: 119-132.

Frost, Mervin. 2009. Global Ethics. Anarchy, Freedom and International Relations. Abingdon: Routledge.

Gallie, William B. 1956. Essentially Contested Concepts. Proceedings of the Aristotelian Society 56: 167-198.

George, Jim. 1994. Discourses of Global Politics. A Critical (Re)Introduction to International Relations. Boulder, CO: Lynne Rienner.

George, Jim. 1995. Realist 'Ethics', International Relations, and Postmodernism: Thinking beyond the Egoism-Anarchy Thematic. Millennium: Journal of International Studies 24: 195-223.

Glynos, Jason, und David Howarth. 2007. Logics of Critical Explanation in Social and Political Theory. London: Routledge.

Goodin, Robert E. 2003. Globalizing Justice. In Taming Globalization. Frontiers of Governance, Hrsg. David Held und Mathias Koenig-Archibugi, 68-92. Cambridge: Polity Press.

Gosepath, Stefan. 2009. Zum Ursprung der Normativität. In Sozialphilosophie und Kritik, Hrsg. Rainer Forst, Martin Hartmann, Rahel Jaeggi und Martin Saar, 250-268. Frankfurt a. M.: Suhrkamp Verlag.

Hahn, Henning. 2009. Globale Gerechtigkeit. Eine philosophische Einführung. Frankfurt a. M./New York: Campus Verlag.

Hanfparade. 2012. Hanfparade 2012 - Freiheit, Gesundheit, Gerechtigkeit! http://www. hanfparade.de/2012.html. Zugegriffen: 08.01.2015.

Hansen, Lene. 2006. Security as Practice. Discourse Analysis and the Bosnian War. London/New York: Routledge.

Henkel, Knuth. 2011. Der Krieg ist außer Kontrolle. Jungle World Online. http://jungleworld.com/artikel/2011/33/43811.html. Zugegriffen: 08.01.2015. 
Herschinger, Eva. 2011. Constructing Global Enemies. Hegemony and Identity in International Discourses on Terrorism and Drug Prohibition. Abingdon/New York: Routledge.

Herschinger, Eva. 2012. 'Hell Is the Other': Conceptualising Hegemony and Identity through Discourse Theory. Millennium - Journal of International Studies 41: 65-90.

Howarth, David, und Yannis Stavrakakis. 2000. Introducing discourse theory and political analysis. In Discourse theory and political analysis. Identities, hegemonies and social change, Hrsg. David Howarth, Yannis Stavrakakis und Aletta J. Norval, 1-23. Manchester: Manchester University Press.

Hudson, Barbara. 2003. Justice in the Risk Society. London: Sage Publications.

Hurrell, Andrew. 2002. Norms and Ethics in International Relations. In Handbook of International Relations, Hrsg. Walter Carlsnaes, Thomas Risse und Beth A. Simmons, 137-154. London/Thousand Oaks/New Delhi: Sage Publications.

Hurrell, Andrew, und Terry MacDonald. 2013. Ethics and Norms in International Relations. In Handbook of International Relations. 2. Auflage, Hrsg. Walter Carlsnaes, Thomas Risse und Beth A. Simmons, 57-84. London: Sage Publications.

International Network of People Who Use Drugs. o. D. INPUD Position Statement on Language, Identity, Inclusivity and Discrimination. Draft version 2.1. http://inpud. wordpress.com/position-papers/inpud-position-statement-on-language-identityinclusivity-and-discrimination/. Zugegriffen: 08.01.2015.

Jabri, Vivienne. 2007. War and the Transformation of Global Politics. Houndsmills: Palgrave Macmillan.

Kearney, Richard. 2003. Strangers, Gods and Monsters. Interpreting Otherness. London: Routledge.

Laclau, Ernesto. 2000a. Foreword. In Discourse theory and political analysis. Identities, hegemonies and social change, Hrsg. David Howarth, Yannis Stavrakakis und Aletta J. Norval, x-xi. Manchester/New York: Manchester University Press.

Laclau, Ernesto. 2000b. Constructing Universality. In Contingency, Hegemony, Universality. Contemporary Dialogues on the Left, Hrsg. Judith Butler, Ernesto Laclau und Slavoj Žižek, 281-307. London: Verso.

Laclau, Ernesto. 2004. Glimpsing the Future. In Laclau. A critical reader, Hrsg. Simon Critchley und Oliver Marchart, 279-328. London/New York: Routledge.

Laclau, Ernesto, und Chantal Mouffe. 2001. Hegemony and Socialist Strategy. Towards a Radical Democratic Politics. London: Verso.

Lawler, Peter. 2008. The Ethics of Postmodernism. In Oxford Handbook of International Relations, Hrsg. Christian Reus-Smit und Duncan Snidal, 378-390. Oxford: Oxford University Press.

Lyon, David. 1999. Postmodernity. 2. Auflage. Buckingham: Open University Press.

Menke, Christoph. 2002. Können und Glauben. Die Möglichkeit der Gerechtigkeit. In Philosophie der Dekonstruktion. Zum Verhältnis von Normativität und Praxis, Hrsg. Andrea Kern und Christoph Menke, 243-263. Frankfurt a. M.: Suhrkamp Verlag.

Methmann, Chris Paul. 2010. 'Climate Protection' as Empty Signifier: A Discourse Theoretical Perspective on Climate Mainstreaming in World Politics. Millennium - Journal of International Studies 39: 345-372.

Miller, David. 2007. National Responsibility and Global Justice. Oxford: Oxford University Press. 
Mission interministérielle de la lutte contre la drogue et la toxicomanie. 1999. Plan triennal de lutte contre la drogue et de prévention des dépendances, 1999-2001: rapport officiel. Paris: La Documentation Française.

Mouffe, Chantal. 1993. The Return of the Political. London/New York: Verso.

Nadelmann, Ethan A. 1990. Global prohibition regimes: the evolution of norms in international society. International Organization 44: 479-526.

NAACP. 2011. NAACP Calls for End to War on Drugs. http://stopthedrugwar.org/ chronicle/2011/jul/26/naacp_calls_end_war_drugs. Zugegriffen: 08.01.2015.

Nonhoff, Martin. 2006. Politischer Diskurs und Hegemonie. Das Projekt „Soziale Marktwirtschaft". Bielefeld: Transcript.

Pin-Fat, Véronique. 2009. How do we begin to think about the world? In Global Politics. A New Introduction, Hrsg. Jenny Edkins und Maja Zehfuss, 22-44. London/New York: Routledge.

Pin-Fat, Véronique. 2010. Universality, Ethics and International Relations. A Grammatical Reading. London: Routledge.

Renner, Judith. 2013. Discourse, Normative Change and the Quest for Reconciliation in Global Politics. Manchester: Manchester University Press.

Sen, Amartya. 2009. The Idea of Justice. Cambridge, MA.: Harvard University Press.

Shapcott, Richard. 2001. Justice, Community and Dialogue in International Relations. Cambridge: Cambridge University Press.

Shapiro, Michael J. 2012. The Micropolitics of Justice: Language, Sense and Space. Law, Culture and the Humanities 8: 466-484.

Smith, Steve. 2004. Singing Our World into Existence: International Relations Theory and September 11. Presidential Address to the International Studies Association, February 27, 2003, Portland. International Studies Quarterly 48: 499-515.

Stäheli, Urs. 1999. Die politische Theorie der Hegemonie: Ernesto Laclau und Chantal Mouffe. In Politische Theorien der Gegenwart, Hrsg. Andre Brodocz und Gary S. Schaal, 141-165. Opladen: Leske + Budrich.

Süddeutsche Zeitung Online. 2013. Papst Franziskus geißelt den Drogenhandel. http:// www.sueddeutsche.de/panorama/rio-de-janeiro-papst-franziskus-geisselt-dendrogenhandel-1.1730194. Zugegriffen: 08.01.2015.

Torfing, Jacob. 1999. New Theories of Discourse: Laclau, Mouffe, and Žižek. Oxford: Blackwell Publishers.

Transnational Institute. 2008. 10 Years. TNI Drugs \& Policy Programme 1998-2008. Amsterdam: Transnational Institute.

United Nations. 1964a. Official Records of United Nations Conference for the Adoption of a Single Convention on Narcotic Drugs, 1961 (Vol. I). New York: United Nations.

United Nations. 1964b. Official Records of United Nations Conference for the Adoption of a Single Convention on Narcotic Drugs, 1961 (Vol. II). New York: United Nations.

United Nations. 1973a. Official Records of United Nations Conference for the Adoption of a Protocol on Psychotropic Substances (Vol. II). New York: United Nations.

United Nations. 1973b. Official Records of United Nations Conference to Consider Amendments to the Single Convention on Narcotic Drugs, 1961 (Vol. II). New York: United Nations. 
United Nations. 1991. Official Records of United Nations Conference for the Adoption of a convention against Illicit Traffic in Narcotic Drugs and Psychotropic Substances (Vol. II). New York: United Nations.

Vaughn-Williams, Nick. 2009. Border Politics: The Limits of Sovereign Power. Edinburgh: Edinburgh University Press.

Vienna NGO Committee on Narcotic Drugs. 2008. „Beyond 2008“ - Declaration and Resolutions. http://www.vngoc.org/details.php?id_cat=13\&id_cnt=59. Zugegriffen: 08.01.2015.

Walker, R.B.J. 1993. Inside/Outside. International Relations as Political Theory. Cambridge: Cambridge University Press.

Walker, R.B.J. 2006. On the Protection of Nature and the Nature of Protection. In The Politics of Protection. Sites of Insecurity and Political Agency, Hrsg. Jef Huysmans, Andrew Dobson und Raia Prokhovnik, 189-203. Abingdon, New York: Routledge.

Walker, R.B.J. 2010. After the Globe, Before the World. London: Routledge.

Wallace, R. Jay. 2011. Konzeptionen der Normativität: Einige grundlegende philosophische Fragen. In Die Herausbildung normativer Ordnungen. Interdisziplinäre Perspektiven, Hrsg. Rainer Forst und Günther Klaus, 33-55. Frankfurt a. M.: Campus Verlag.

Welch, David. 1993. Justice and the Genesis of War. Cambridge: Cambridge University Press.

White, Stephen K. 1988. Poststructuralism and Political Reflection. Political Theory 6: 186-208.

Zehfuss, Maja. 2007. Subjectivity and Vulnerability: On the War with Iraq. International Politics 44: 58-71. DOI: 10.1057/palgrave.ip.8800158.

Zehfuss, Maja. 2011. Targeting: Precision and the production of ethics. European Journal of International Relations 17: 543-566.

Zehfuss, Maja. 2013. Critical Theory, Poststructuralism, and Postcolonialism. In Handbook of International Relations. 2. Auflage, Hrsg. Walter Carlsnaes, Thomas Risse und Beth A. Simmons, 145-169. Los Angeles: Sage Publications.

ZIB Forum. 2010. Internationale Politische Theorie. Zeitschrift für Internationale Beziebungen 17: 267-363.

\section{Autorenangaben}

Dr. Eva Herschinger,

Lecturer in Politics and International Relations,

University of Aberdeen, Department of Politics and International Relations,

Edward Wright Building, Aberdeen, AB24 3QY,

Großbritannien, eva.herschinger@abdn.ac.uk. 